\title{
Face-Centered Cubic Refractory Alloys Prepared from Single-Source Precursors
}

\author{
Kirill V. Yusenko ${ }^{1, *(\mathbb{D}}$, Saiana Khandarkhaeva ${ }^{2}$, Maxim Bykov ${ }^{2}{ }^{\mathbb{D}}$, Tymofey Fedotenko ${ }^{2}$, \\ Michael Hanfland ${ }^{3}$, Alexander Sukhikh ${ }^{4}{ }^{\mathbb{D}}$, Sergey A. Gromilov ${ }^{4}$ and Leonid S. Dubrovinsky ${ }^{2}$ \\ 1 BAM Federal Institute for Materials Research and Testing, Richard-Willstätter Str. 11, D-12489 Berlin, Germany \\ 2 Bayerisches Geoinstitut, Universität Bayreuth, D-95440 Bayreuth, Germany; \\ Saiana.Khandarkhaeva@uni-bayreuth.de (S.K.); maks.byk@gmail.com (M.B.); \\ timofeyfedotenko@gmail.com (T.F.); Leonid.Dubrovinsky@uni-bayreuth.de (L.S.D.) \\ 3 ESRF - The European Synchrotron 71 Avenue des Martyrs, 38000 Grenoble, France; hanfland@esrf.fr \\ 4 Nikolaev Institute of Inorganic Chemistry, Lavrentiev ave. 3, 630090 Novosibirsk, Russia; \\ a_sukhikh@niic.nsc.ru (A.S.); grom@niic.nsc.ru (S.A.G.) \\ * Correspondence: kirill.yusenko@bam.de
}

Received: 9 January 2020; Accepted: 13 March 2020; Published: 20 March 2020

\begin{abstract}
Three binary $f c c$-structured alloys $\left(f c c-\mathrm{Ir}_{0.50} \mathrm{Pt}_{0.50}, f c c-\mathrm{Rh}_{0.66} \mathrm{Pt}_{0.33}\right.$ and $\left.f c c-\mathrm{Rh}_{0.50} \mathrm{Pd}_{0.50}\right)$ were prepared from $\left[\operatorname{Ir}\left(\mathrm{NH}_{3}\right)_{5} \mathrm{Cl}\right]\left[\mathrm{PtCl}_{6}\right], \quad\left[\mathrm{Ir}\left(\mathrm{NH}_{3}\right)_{5} \mathrm{Cl}\right]\left[\mathrm{PtBr}_{6}\right], \quad\left[\mathrm{Rh}\left(\mathrm{NH}_{3}\right)_{5} \mathrm{Cl}_{2}\left[\mathrm{PtCl}_{6}\right] \mathrm{Cl}_{2}\right.$ and $\left[\mathrm{Rh}\left(\mathrm{NH}_{3}\right)_{5} \mathrm{Cl}\right]\left[\mathrm{PdCl}_{4}\right] \cdot \mathrm{H}_{2} \mathrm{O}$, respectively, as single-source precursors. All alloys were prepared by thermal decomposition in gaseous hydrogen flow below $800{ }^{\circ} \mathrm{C}$. $F c c-\mathrm{Ir}_{0.50} \mathrm{Pt}_{0.50}$ and $f c c-\mathrm{Rh}_{0.50} \mathrm{Pd}_{0.50}$ correspond to miscibility gaps on binary metallic phase diagrams and can be considered as metastable alloys. Detailed comparison of $\left[\operatorname{Ir}\left(\mathrm{NH}_{3}\right)_{5} \mathrm{Cl}\right]\left[\mathrm{PtCl}_{6}\right]$ and $\left[\operatorname{Ir}\left(\mathrm{NH}_{3}\right)_{5} \mathrm{Cl}\right]\left[\mathrm{PtBr}_{6}\right]$ crystal structures suggests that two isoformular salts are not isostructural. $\operatorname{In}\left[\operatorname{Ir}\left(\mathrm{NH}_{3}\right)_{5} \mathrm{Cl}\right]\left[\mathrm{PtBr}_{6}\right]$, specific $\mathrm{Br} \ldots \mathrm{Br}$ interactions are responsible for a crystal structure arrangement. Room temperature compressibility of $f c c-\mathrm{Ir}_{0.50} \mathrm{Pt}_{0.50}, f c c-\mathrm{Rh}_{0.66} \mathrm{Pt}_{0.33}$ and $f c c-\mathrm{Rh}_{0.50} \mathrm{Pd}_{0.50}$ has been investigated up to 50 GPa in diamond anvil cells. All investigated $f c c$-structured binary alloys are stable under compression. Atomic volumes and bulk moduli show good agreement with ideal solutions model. For $f c c-\mathrm{Ir}_{0.50} \mathrm{Pt}_{0.50}$, $V_{0} / Z=14.597(6) \AA^{3} \cdot$ atom $^{-1}, B_{0}=321(6) \mathrm{GPa}$ and $B_{0}{ }^{\prime}=6(1)$; for $f c c-\mathrm{Rh}_{0.66} \mathrm{Pt}_{0.33}, V_{0} / Z=14.211(3)$ $\AA^{3} \cdot$ atom $^{-1}, B_{0}=259(1) \mathrm{GPa}$ and $B_{0}{ }^{\prime}=6.66(9)$ and for $f c c-\mathrm{Rh}_{0.50} \mathrm{Pd}_{0.50}, V_{0} / Z=14.18(2) \AA^{3} \cdot$ atom $^{-1}$, $B_{0}=223(4) \mathrm{GPa}$ and $B_{0}{ }^{\prime}=5.0(3)$.
\end{abstract}

Keywords: refractory alloys; platinum group metals; single-source precursors; high-pressure

\section{Introduction}

Traditionally, high-entropy alloys were prepared using conventional melting of pure metals. Nevertheless, catalytic applications as well as preparation of high-entropy alloys based on metals with ultra-high melting points need the development of new techniques for a preparation of high-entropy alloys as fine nanostructured powders. Recently, single-source precursors strategy has been applied to access high-entropy alloys based on platinum group metals. The strategy requires a synthesis of coordination compounds from water solutions and their further thermal decomposition in a hydrogen flow based on the following principle general scheme [1]:

$$
\begin{gathered}
\text { in water solution: } a\left[\operatorname{Ir}\left(\mathrm{NH}_{3}\right)_{5} \mathrm{Cl}\right] \mathrm{Cl}_{2}+b\left[\mathrm{Rh}\left(\mathrm{NH}_{3}\right)_{5} \mathrm{Cl}_{C} \mathrm{Cl}_{2}+(1-a-b)\left[\mathrm{Ru}\left(\mathrm{NH}_{3}\right)_{5} \mathrm{Cl}\right] \mathrm{Cl}_{2}+\right. \\
c\left(\mathrm{NH}_{4}\right)_{2}\left[\mathrm{IrCl}_{6}\right]+d\left(\mathrm{NH}_{4}\right)_{2}\left[\mathrm{OsCl}_{6}\right]+e\left(\mathrm{NH}_{4}\right)_{2}\left[\mathrm{PtCl}_{6}\right]+(1-c-d-e)\left(\mathrm{NH}_{4}\right)_{2}\left[\mathrm{ReCl}_{6}\right] \rightarrow \\
{\left[\mathrm{Ir}\left(\mathrm{NH}_{3}\right)_{5} \mathrm{Cl}\right]_{a}\left[\mathrm{Rh}\left(\mathrm{NH}_{3}\right)_{5} \mathrm{Cl}\right]_{b}\left[\mathrm{Ru}\left(\mathrm{NH}_{3}\right)_{5} \mathrm{Cl}\right]_{(1-a-b)}\left[\mathrm{IrCl}_{6}\right]_{c}\left[\mathrm{OsCl}_{6}\right]_{d}\left[\mathrm{PtCl}_{6}\right]_{e}\left[\mathrm{ReCl}_{6}\right]_{(1-c-d-e)}+2 \mathrm{NH}_{4} \mathrm{Cl}}
\end{gathered}
$$


in solid state: $\left[\operatorname{Ir}\left(\mathrm{NH}_{3}\right)_{5} \mathrm{Cl}\right]_{a}\left[\mathrm{Rh}\left(\mathrm{NH}_{3}\right)_{5} \mathrm{Cl}\right]_{b}\left[\mathrm{Ru}\left(\mathrm{NH}_{3}\right)_{5} \mathrm{Cl}\right]_{(1-a-b)}\left[\operatorname{IrCl}_{6}\right]_{c}\left[\mathrm{OsCl}_{6}\right]_{d}\left[\mathrm{PtCl}_{6}\right]_{e}\left[\mathrm{ReCl}_{6}\right]_{(1-c-d-e)}$ $+7 / 2 \mathrm{H}_{2(\text { gas })} \rightarrow \mathrm{Ir}_{0.5(a+c)} \mathrm{Rh}_{0.5 b} \mathrm{Ru}_{0.5(1-a-b)} \mathrm{Os}_{0.5 d} \mathrm{Pt}_{0.5 e} \mathrm{Re}_{0.5(1-c-d-e)}+5 \mathrm{NH}_{4} \mathrm{Cl}_{(\text {gas })}+2 \mathrm{HCl}_{(\text {gas })}$.

Several two phases as well as $h c p-\operatorname{Ir}_{0.19} \mathrm{Os}_{0.22} \operatorname{Re}_{0.21} \mathrm{Rh}_{0.20} \mathrm{Ru}_{0.19}$ and $f c c-\operatorname{Ir}_{0.26} \mathrm{Os}_{0.05} \mathrm{Pt}_{0.31} \mathrm{Rh}_{0.23} \mathrm{Ru}_{0.15}$ single-phase high-entropy alloys were prepared using the single-source precursors strategy [1]. The approach is quite general and can be extended to access refractory high-entropy alloys in a broad compositional range. Single-phase $f c c$ - and $h c p$-structured high-entropy alloys were also tested under extreme conditions to characterize their pressure and temperature stability. Nevertheless, due to their compositional complexity, a little was investigated regarding a mechanism of their formation from single source precursors.

Refractory multicomponent alloys such as platinum group alloys attract attention as catalytically active species with high mechanical and chemical stability. Ir- and Rh-based alloys were proposed as materials for high-temperature applications, as thermocouples and crucibles. Refractory alloys usually have also high stability under extreme conditions and show low compressibility. Their low compressibility might be compared only with diamond [2-4]. Previously, several binary and multicomponent refractory systems of $f c c$ - and $h c p$-structured alloys were investigated under extreme conditions [5-8]. It has been shown that all known platinum group alloys do not show any temperature and/or pressure induced phase transitions. Such a finding makes platinum group alloys important as stable materials with a unique phase and structural stability under extreme conditions. Ultra-incompressible alloys with Re, Os $(h c p)$ and Ir $(f c c)$ were investigated in detail up to $140 \mathrm{GPa}$; nevertheless, compressibility of $f c c$-structured alloys of metals with high compressibility such as $\mathrm{Rh}$ and $\mathrm{Pd}$ were not investigated so far. Among $f c c$-structured alloys, only $f c c-\mathrm{Ir}_{0.42} \mathrm{Rh}_{0.58}$ has been investigated up to $57 \mathrm{GPa}$ at room temperature [5].

In the current study, we report synthesis of $f c c-\mathrm{Ir}_{0.50} \mathrm{Pt}_{0.50}, f c c-\mathrm{Rh}_{0.66} \mathrm{Pt}_{0.33}$ and $f c c-\mathrm{Rh}_{0.50} \mathrm{Pd}_{0.50}$ binary alloys from $\left[\operatorname{Ir}\left(\mathrm{NH}_{3}\right)_{5} \mathrm{Cl}\right]\left[\mathrm{PtCl}_{6}\right], \quad\left[\operatorname{Ir}\left(\mathrm{NH}_{3}\right)_{5} \mathrm{Cl}\right]\left[\mathrm{PtBr}_{6}\right], \quad\left[\mathrm{Rh}\left(\mathrm{NH}_{3}\right)_{5} \mathrm{Cl}_{2}\left[\mathrm{PtCl}_{6}\right] \mathrm{Cl}_{2}\right.$ and $\left[\mathrm{Rh}\left(\mathrm{NH}_{3}\right)_{5} \mathrm{Cl}\right]\left[\mathrm{PdCl}_{4}\right] \cdot \mathrm{H}_{2} \mathrm{O}$, respectively, as single-source precursors under low temperature. Investigation of $f c c-\mathrm{Ir}_{0.50} \mathrm{Pt}_{0.50}, f c c-\mathrm{Rh}_{0.66} \mathrm{Pt}_{0.33}$ and $f c c-\mathrm{Rh}_{0.50} \mathrm{Pd}_{0.50}$ binary alloys under hydrostatic compression up to $50 \mathrm{GPa}$ in diamond anvil cells allow us to obtain bulk moduli for $f c c$-structured binary alloys. Equations of state, thermal expansion and pressure compressibility for known refractory high-entropy alloys can be validated with experimental data obtained for $f c c$-structured refractory binaries.

\section{Materials and Methods}

$\left[\operatorname{Ir}\left(\mathrm{NH}_{3}\right)_{5} \mathrm{Cl}\right] \mathrm{Cl}_{2}$ and $\left[\mathrm{Rh}\left(\mathrm{NH}_{3}\right)_{5} \mathrm{Cl}_{\mathrm{Cl}} \mathrm{Cl}_{2}\right.$ were prepared from $\mathrm{IrCl}_{4} \cdot x \mathrm{H}_{2} \mathrm{O}$ and $\mathrm{RhCl}_{3} \cdot x \mathrm{H}_{2} \mathrm{O}$ according to published protocols $[9,10]$. $\left(\mathrm{NH}_{4}\right)_{2}\left[\mathrm{PtCl}_{6}\right],\left(\mathrm{NH}_{4}\right)_{2}\left[\mathrm{PdCl}_{4}\right]$ and $\left(\mathrm{NH}_{4}\right)_{2}\left[\mathrm{IrCl}_{6}\right]$ were obtained from (Sigma Aldrich). Binary alloys $f c c-\operatorname{Ir}_{0.50} \mathrm{Pt}_{0.50}$ (sample A), $f c c-\mathrm{Rh}_{0.66} \mathrm{Pt}_{0.33}$ (sample B) and $f c c-\mathrm{Rh}_{0.50} \mathrm{Pd}_{0.50}$ (sample C) were prepared from $\left[\operatorname{Ir}\left(\mathrm{NH}_{3}\right)_{5} \mathrm{Cl}\right]\left[\mathrm{PtCl} \mathrm{C}_{6}\right],\left[\mathrm{Rh}\left(\mathrm{NH}_{3}\right)_{5} \mathrm{Cl}_{2}\left[\mathrm{PtCl}_{6}\right] \mathrm{Cl}_{2}\right.$ and $\left[\mathrm{Rh}\left(\mathrm{NH}_{3}\right)_{5} \mathrm{Cl}\right]\left[\mathrm{PdCl}_{4}\right] \cdot \mathrm{H}_{2} \mathrm{O}$ respectively. Details about the synthesis of solid-state precursors and alloys can be found in our earlier publications $[8,10]$. Briefly, the precursors, $\left[\operatorname{Ir}\left(\mathrm{NH}_{3}\right)_{5} \mathrm{Cl}\right]\left[\mathrm{PtCl}_{6}\right]$, was first crystallized at room temperature from a mixture of water solutions of $\left[\operatorname{Ir}\left(\mathrm{NH}_{3}\right)_{5} \mathrm{Cl}\right] \mathrm{Cl}_{2}$ and $\left(\mathrm{NH}_{4}\right)_{2}\left[\mathrm{PtCl}_{6}\right]$, filtered and dried on air. The obtained solid powder of $\left[\operatorname{Ir}\left(\mathrm{NH}_{3}\right)_{5} \mathrm{Cl}\right]\left[\mathrm{PtCl}_{6}\right]$ was then heated in the hydrogen flow up to $700{ }^{\circ} \mathrm{C}$ and cooled down to ambient temperature. Similarly, $\left[\mathrm{Rh}\left(\mathrm{NH}_{3}\right)_{5} \mathrm{Cl}\right]\left[\mathrm{PdCl}_{4}\right] \cdot \mathrm{H}_{2} \mathrm{O}$ was prepared at room temperature from $\left[\mathrm{Rh}\left(\mathrm{NH}_{3}\right)_{5} \mathrm{Cl} \mathrm{Cl}_{2}\right.$ and $\left(\mathrm{NH}_{4}\right)_{2}\left[\mathrm{PdCl}_{4}\right]$ and heated in hydrogen flow to $400{ }^{\circ} \mathrm{C}$ and cooled to ambient temperature during $2 \mathrm{~h}$. $\left[\mathrm{Rh}\left(\mathrm{NH}_{3}\right)_{5} \mathrm{Cl}\right]_{2}\left[\mathrm{PtCl}_{6}\right] \mathrm{Cl}_{2}$ was prepared from water solution of $\left(\mathrm{NH}_{4}\right)_{2}\left[\mathrm{PtCl}_{6}\right]$ in $0.1 \mathrm{M} \mathrm{HCl}$ and solid $\left[\mathrm{Rh}\left(\mathrm{NH}_{3}\right)_{5} \mathrm{Cl}\right] \mathrm{Cl}_{2}$. The mixture was kept in darkness at room temperature for a week. Orange crystals were filtered and dried on air. $\left[\mathrm{Rh}\left(\mathrm{NH}_{3}\right)_{5} \mathrm{Cl}_{2}\left[\mathrm{PtCl}_{6}\right] \mathrm{Cl}_{2}\right.$ crystals were decomposed in hydrogen flow at $700^{\circ} \mathrm{C}$. Similarly, all compounds were also decomposed in He flow. All preparatory conditions for all alloys are summarized in Table 1. 
Table 1. Preparatory conditions and crystallographic characteristics for $f c c$-structured $\mathrm{Rh}, \mathrm{Ir}, \mathrm{Pt}$ and $\mathrm{Pd}$ binary alloys.

\begin{tabular}{|c|c|c|c|c|c|}
\hline $\begin{array}{c}\text { Single-Source Precursor } \\
\text { Ref. }\end{array}$ & $\begin{array}{l}\text { Preparatory } \\
\text { Conditions }\end{array}$ & $\begin{array}{c}\text { Phase } \\
\text { Composition }\end{array}$ & $a, \AA^{*}$ & $\begin{array}{c}V / Z, \\
\AA^{3} \cdot \text { atom }^{-1}\end{array}$ & $\Delta a, \AA$ \\
\hline \multirow[t]{2}{*}[\mathrm{Ir}(\mathrm{NH}_{3})_{5}\mathrm{Cl}]{$\left[\mathrm{PtCl}_{6}\right]$} & He flow, $400^{\circ} \mathrm{C}$ & two $f c c$ phases & $\begin{array}{l}a_{I}=3.919(6) \\
a_{I I}=3.847(6)\end{array}$ & - & - \\
\hline & $\mathrm{H}_{2}$ flow, $700^{\circ} \mathrm{C}$ & $\begin{array}{c}f c c-\operatorname{Ir}_{0.50} \mathrm{Pt}_{0.50} \\
\text { sample } \mathbf{A}\end{array}$ & $3.883(2)$ & 14.537 & -0.08 \\
\hline \multirow{2}{*}[\operatorname{Ir}(\mathrm{NH}_{3})_{5}\mathrm{Cl}]{$\left[\mathrm{PtBr}_{6}\right]$} & He flow, $600^{\circ} \mathrm{C}$ & two $f c c$ phases & - & - & - \\
\hline & $\mathrm{H}_{2}$ flow, $500^{\circ} \mathrm{C}$ & $f_{c c}-\mathrm{Ir}_{0.50} \mathrm{Pt}_{0.50}$ & $3.874(2)$ & 14.535 & -0.085 \\
\hline \multirow{2}{*}{$\begin{array}{c}{\left[\mathrm{Ir}\left(\mathrm{NH}_{3}\right)_{5} \mathrm{Cl}_{2}\left[\mathrm{PtCl}_{6}\right] \mathrm{Cl}_{2}\right.} \\
\text { [11] (only crystal structure was } \\
\text { reported) }\end{array}$} & He flow, $460^{\circ} \mathrm{C}$ & two $f_{c c}$ phases & $\begin{array}{l}a_{I}=3.855 \\
a_{I I}=3.917\end{array}$ & - & - \\
\hline & $\mathrm{H}_{2}$ flow, $500{ }^{\circ} \mathrm{C}$ & $f c c-\operatorname{Ir}_{0.67} \mathrm{Pt}_{0.33}$ & $3.870(1)$ & 14.490 & 0.03 \\
\hline \multirow[t]{2}{*}[\mathrm{Rh}(\mathrm{NH}_{3})_{5}\mathrm{Cl}]{$\left[\mathrm{PtCl}_{6}\right]$} & He flow, $600^{\circ} \mathrm{C}$ & $\begin{array}{l}f c c-\mathrm{Rh}_{0.72} \mathrm{Pt}_{0.28} \\
f c c-\mathrm{Rh}_{0.79} \mathrm{Pt}_{0.21}\end{array}$ & $\begin{array}{l}a_{I}=3.837(4) \\
a_{I I}=3.828(4)\end{array}$ & - & - \\
\hline & $\mathrm{H}_{2}$ flow, $550^{\circ} \mathrm{C}$ & $f c c-\mathrm{Rh}_{0.5} \mathrm{Pt}_{0.5}$ & $3.865(4)$ & 14.434 & 0.002 \\
\hline \multirow[t]{2}{*}[\mathrm{Rh}(\mathrm{NH}_{3})_{5}\mathrm{Cl}]{$\left[\mathrm{PtBr}_{6}\right][12]$} & He flow, $800^{\circ} \mathrm{C}$ & $\begin{array}{l}f_{c c-}-\mathrm{Rh}_{0.38} \mathrm{Pt}_{0.62} \\
f c c-\mathrm{Rh}_{0.72} \mathrm{Pt}_{0.28}\end{array}$ & $\begin{array}{l}a_{I}=3.878(3) \\
a_{I I}=3.836(3)\end{array}$ & - & - \\
\hline & $\mathrm{H}_{2}$ flow, $700^{\circ} \mathrm{C}$ & $f_{c c}-\mathrm{Rh}_{0.50} \mathrm{Pt}_{0.50}$ & $3.864(2)$ & 14.423 & 0.001 \\
\hline \multirow{2}{*}{$\begin{array}{c}{\left[\mathrm{Rh}\left(\mathrm{NH}_{3}\right)_{5} \mathrm{Cl}\right]_{2}\left[\mathrm{PtCl}_{6}\right] \mathrm{Cl}_{2}} \\
{[13]}\end{array}$} & He flow, $460^{\circ} \mathrm{C}$ & $\begin{array}{l}f c c-\mathrm{Rh}_{0.03} \mathrm{Pt}_{0.97} \\
f c c-\mathrm{Rh}_{0.93} \mathrm{Pt}_{0.07}\end{array}$ & $\begin{aligned} a_{I} & =3.919(5) \\
a_{I I} & =3.811(5)\end{aligned}$ & - & - \\
\hline & $\mathrm{H}_{2}$ flow, $500{ }^{\circ} \mathrm{C}$ & $\begin{array}{c}f_{c c-}-\mathrm{Rh}_{0.66} \mathrm{Pt}_{0.33} \\
\text { sample B }\end{array}$ & $3.845(5)$ & 14.211 & 0.001 \\
\hline \multirow{2}{*}{$\begin{array}{c}{\left[\mathrm{Rh}\left(\mathrm{NH}_{3}\right)_{5} \mathrm{Cl}\right]\left[\mathrm{IrCl}_{6}\right]} \\
{[5,14]}\end{array}$} & Ar flow, $550^{\circ} \mathrm{C}$ & $f c c-\mathrm{Rh}_{0.50} \mathrm{Ir}_{0.50}$ & $3.817(2)$ & 13.903 & -0.004 \\
\hline & $\mathrm{H}_{2}$ flow $650^{\circ} \mathrm{C}$ & $f c c-\mathrm{Rh}_{0.50} \mathrm{Ir}_{0.50}$ & $3.825(2)$ & 13.991 & 0.004 \\
\hline \multirow{2}{*}[\mathrm{Rh}(\mathrm{NH}_{3})_{5}\mathrm{Cl}]{$\left[\mathrm{IrBr}_{6}\right][15]$} & He flow, $800^{\circ} \mathrm{C}$ & $f_{c c}-\mathrm{Rh}_{0.50} \mathrm{Ir}_{0.50}$ & $3.820(2)$ & 13.936 & 0.001 \\
\hline & $\mathrm{H}_{2}$ flow, $600^{\circ} \mathrm{C}$ & $f_{c c}-\mathrm{Rh}_{0.50} \mathrm{Ir}_{0.50}$ & $3.824(2)$ & 13.980 & 0.003 \\
\hline \multirow{2}{*}[\mathrm{Rh}(\mathrm{NH}_{3})_{5}\mathrm{Cl}]{$_{2}\left[\mathrm{IrCl}_{6}\right] \mathrm{Cl}_{2}[16]$} & He flow, $470^{\circ} \mathrm{C}$ & $f c c-\mathrm{Rh}_{0.66} \mathrm{Ir}_{0.33}$ & $3.810(2)$ & 13.827 & -0.005 \\
\hline & $\mathrm{H}_{2}$ flow, $700^{\circ} \mathrm{C}$ & $f c c-\mathrm{Rh}_{0.66} \mathrm{Ir}_{0.33}$ & $3.813(4)$ & 13.859 & -0.002 \\
\hline$\left[\mathrm{Rh}\left(\mathrm{NH}_{3}\right)_{5} \mathrm{Cl}\right]\left[\mathrm{PdCl}_{4}\right] \cdot \mathrm{H}_{2} \mathrm{O}[9]$ & $\mathrm{H}_{2}$ flow, $400^{\circ} \mathrm{C}$ & $\begin{array}{l}f_{c c-}-\mathrm{Rh}_{0.50} \mathrm{Pd}_{0.50} \\
\text { sample C }\end{array}$ & $3.845(4)$ & 14.211 & -0.002 \\
\hline
\end{tabular}

* Cell parameters for pure metals: $a(\mathrm{Pt})=3.9231 \AA\left(V / Z=15.095 \AA^{3}\right) ; a(\mathrm{Ir})=3.8394 \AA\left(V / Z=14.145 \AA^{3}\right) ; a(\mathrm{Rh})=$ $3.8031 \AA\left(V / Z=13.750 \AA^{3}\right)$ and $a(\mathrm{Pd})=3.8898 \AA\left(V / Z=13.819 \AA^{3}\right)$.

Two isoformular salts, $\left[\mathrm{Ir}\left(\mathrm{NH}_{3}\right)_{5} \mathrm{Cl}\right]\left[\mathrm{PtCl}_{6}\right]$ and $\left[\mathrm{Ir}\left(\mathrm{NH}_{3}\right)_{5} \mathrm{Cl}\right]\left[\mathrm{PtBr}_{6}\right]$, were prepared by the mixing of hot water solutions of $\left[\mathrm{Ir}\left(\mathrm{NH}_{3}\right)_{5} \mathrm{Cl}\right] \mathrm{Cl}_{2}$ and $\left(\mathrm{NH}_{4}\right)_{2}\left[\mathrm{PtCl}_{6}\right]$ or $\left(\mathrm{NH}_{4}\right)_{2}\left[\mathrm{PtBr}_{6}\right]$, respectively. After 40-60 min, orange precipitate of $\left[\operatorname{Ir}\left(\mathrm{NH}_{3}\right)_{5} \mathrm{Cl}\right]\left[\mathrm{PtCl}_{6}\right]$ and orange-red precipitate of $\left[\operatorname{Ir}\left(\mathrm{NH}_{3}\right)_{5} \mathrm{Cl}\right]\left[\mathrm{PtBr}_{6}\right]$ were filtered, washed with minimum water, ethanol and dried on air. Red plate-shaped single crystals of $\left[\mathrm{Ir}\left(\mathrm{NH}_{3}\right)_{5} \mathrm{Cl}\right]\left[\mathrm{PtBr}_{6}\right]$ were collected from the whole portion of the sample. Yield for $\left[\operatorname{Ir}\left(\mathrm{NH}_{3}\right)_{5} \mathrm{Cl}\right]\left[\mathrm{PtCl}_{6}\right]$ was $75 \%-80 \%$ and yield for $\left[\operatorname{Ir}\left(\mathrm{NH}_{3}\right)_{5} \mathrm{Cl}\right]\left[\mathrm{PtBr}_{6}\right]$ was $85 \%-90 \%$.

Elemental analysis:

\begin{tabular}{ccc}
\hline Composition & Ir+Pt, wt \%, Calculated & Ir+Pt, wt \%, Obtained \\
\hline$\left[\operatorname{Ir}\left(\mathrm{NH}_{3}\right)_{5} \mathrm{Cl}\right]\left[\mathrm{PtBr}_{6}\right]$ & 39.23 & $40.3 \pm 0.2$ \\
{$\left[\operatorname{Ir}\left(\mathrm{NH}_{3}\right)_{5} \mathrm{Cl}\right]\left[\mathrm{PtCl}_{6}\right]$} & 53.75 & $53.3 \pm 0.2$ \\
\hline
\end{tabular}

Thermal analysis of $\left[\operatorname{Ir}\left(\mathrm{NH}_{3}\right)_{5} \mathrm{Cl}\right]\left[\mathrm{PtCl}_{6}\right]$ and $\left[\mathrm{Ir}\left(\mathrm{NH}_{3}\right)_{5} \mathrm{Cl}\right]\left[\mathrm{PtBr}_{6}\right]$ was performed on a Q-1000 TG device. Powders (ca. $0.1 \mathrm{~g}$ ) were heated in Pt crucibles closed with a lead. Heating $(5 \mathrm{~K} / \mathrm{min}$ ) was performed in helium flow (150 mL/min). $\mathrm{Al}_{2} \mathrm{O}_{3}$ has been used as a reference. $\left[\operatorname{Ir}\left(\mathrm{NH}_{3}\right)_{5} \mathrm{Cl}\right]\left[\mathrm{PtCl}_{6}\right]$ decomposes in a narrow temperature interval $325-425^{\circ} \mathrm{C}$; $\left[\mathrm{Ir}\left(\mathrm{NH}_{3}\right)_{5} \mathrm{Cl}\right]\left[\mathrm{PtBr}_{6}\right]$ decomposes between 330 and $530{ }^{\circ} \mathrm{C}$ (Figure 1). 


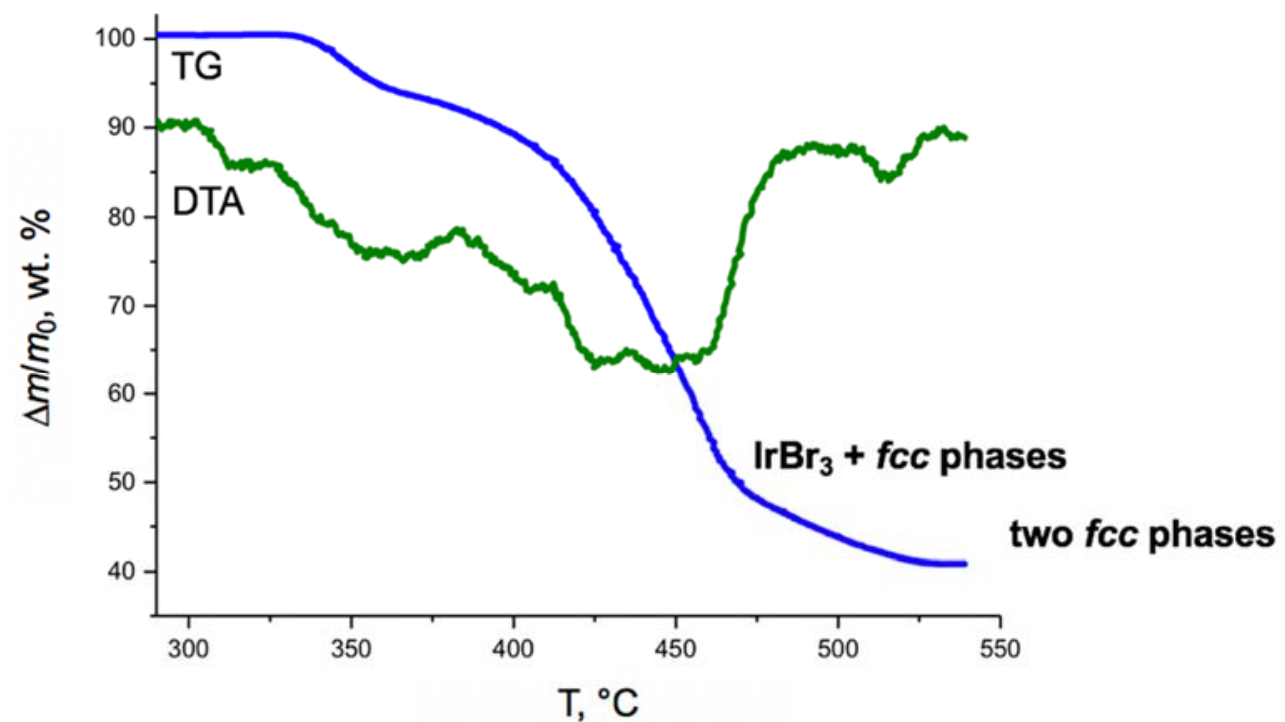

Figure 1. Thermal gravimetry (TG) and differential thermal analysis (DTA) curves for $\left[\operatorname{Ir}\left(\mathrm{NH}_{3}\right)_{5} \mathrm{Cl}\right]\left[\mathrm{PtBr}_{6}\right]$ in helium flow (closed Pt crucible, $5 \mathrm{~K} / \mathrm{min}, 150 \mathrm{~mL} / \mathrm{min}$ ).

Phase composition and cell parameters of metallic alloys were obtained by in house powder X-ray diffraction (PXRD) using an ARL X'TRA diffractometer $(\mathrm{CuK} \alpha$-radiation, Ni-filter, position sensitive detectors, Bragg-Brentano reflection geometry, $2 \Theta=5-100^{\circ}, \Delta 2 \Theta=0.03^{\circ}, 10 \mathrm{~s} / \mathrm{step}$, room temperature, Thermo Electron Corporation, Waltham, MA, USA). Polycrystalline samples were slightly ground with hexane using an agate mortar, and the resulting suspensions were deposited on the polished side of a quartz sample holder, a smooth thin layer formed after drying. Silicon powder was taken as an external standard $\left(a=5.4309 \AA\right.$, full width at half maximum $\left.2 \Theta=0.1^{\circ}\right)$ for the calibration of the zero-shift of the goniometer and instrumental line broadening. Only single- and two-phase $f c c$-structured alloys were found as products of thermal decomposition of single-source precursors mentioned above (Table 1).

Scanning electron microscopy (SEM) images for $f c c-\mathrm{Ir}_{0.50} \mathrm{Pt}_{0.50}, f c c-\mathrm{Rh}_{0.66} \mathrm{Pt}_{0.33}$ and $f c c-\mathrm{Rh}_{0.50} \mathrm{Pd}_{0.50}$ binary alloys prepared in hydrogen atmosphere were obtained on XL30 ESEM (Environmental Scanning Electron Microscope) from FEI (Thermo Fisher Scientific, Waltham, Massachusetts, USA). The composition was obtained by energy-dispersive X-ray analysis (EDAX, equipped with Si-(Li) detector) and averaged for 5-6 points. The averaged composition was close to the nominal composition of single-source precursors. So, $f c c-\mathrm{Ir}_{0.509(5)} \mathrm{Pt}_{0.491(5)}$ correspond to $f c c-\mathrm{Ir}_{0.50} \mathrm{Pt}_{0.50}$ (sample A); $f c c-\mathrm{Rh}_{0.657(4)} \mathrm{Pt}_{0.343(4)}$ correspond to $f c c-\mathrm{Rh}_{0.66} \mathrm{Pt}_{0.33}$ (sample B); and $f c c-\mathrm{Rh}_{0.497(5)} \mathrm{Pd}_{0.502(5)}$ correspond to $f c c-\mathrm{Rh}_{0.50} \mathrm{Pd}_{0.50}$ (sample $\mathrm{C}$ ).

Room temperature compressibility curves for $f c c-\mathrm{Ir}_{0.50} \mathrm{Pt}_{0.50}, f c c-\mathrm{Rh}_{0.66} \mathrm{Pt}_{0.33}$ and $f c c-\mathrm{Rh}_{0.50} \mathrm{Pd}_{0.50}$ binary alloys were collected at the ID15B beamline up to 40 GPa (ESRF, $\lambda=0.411235 \AA$, MAR 555 flat panel detector, beam size $10(v) \times 10(h) \mu \mathrm{m}^{2}$ ). The samples were loaded in diamond anvil cells equipped with conically supported Boehler Almax anvils $250 \mu \mathrm{m}$ culet sizes. He was used as a pressure transmitting medium. Ruby was applied as a pressure calibrant. The diffraction data were integrated using DIOPTAS [17]. The unit cell parameters, the background and the line-profile parameters were refined simultaneously using JANA2006 software [18]. The $P-V$ data were fitted using EoS-Fit 5.2 software [19].

The X-ray diffraction study of $\left[\operatorname{Ir}\left(\mathrm{NH}_{3}\right)_{5} \mathrm{Cl}\right]\left[\mathrm{PtBr}_{6}\right]$ single crystal at $150 \mathrm{~K}$ was performed on an automated Bruker APEX-II CCD diffractometer (MoK $\alpha$ radiation, graphite monochromator, two-dimensional CCD detector, Bruker Corporation, Karlsruhe, Germany). One hundred and fifty nine structural parameters with 48 restrains were refined and 4159 reflexes were used. The corresponding divergence factors were $R_{\text {all }}=9.07 \%$ and $w R_{\text {ref }}=15.41 \%$; for 3150 reflections with $I \geq 2 \sigma(I), R_{\mathrm{gt}}=6.28 \%$, $w R_{\mathrm{gt}}=14.33 \%$ and the $S$ factor against $F_{2}$ was 1.066 . X-ray crystallographic data were deposited with an Inorganic Crystal Structure Database (ICSD) under No. 1971298. 


\section{Results and Discussion}

\subsection{Crystal Structures of $\left[\operatorname{Ir}\left(\mathrm{NH}_{3}\right)_{5} \mathrm{Cl}\right]\left[\mathrm{PtCl}_{6}\right]$ and $\left[\mathrm{Ir}\left(\mathrm{NH}_{3}\right)_{5} \mathrm{Cl}\right]\left[\mathrm{PtBr}{ }_{6}\right]$ Single-Source Precursors}

Synthesis and crystal structures for isoformular $\left[M^{\mathrm{I}}\left(\mathrm{NH}_{3}\right)_{5} \mathrm{Cl}\right]_{2}\left[\mathrm{M}^{\mathrm{II}} \mathrm{Cl}_{6}\right] \mathrm{Cl}_{2}$ compounds where $M^{\mathrm{I}}$ $=\mathrm{Co}, \mathrm{Cr}, \mathrm{Ir}, \mathrm{Rh}, \mathrm{Os}$ and $M^{\mathrm{II}}=\mathrm{Ir}, \mathrm{Pt}, \mathrm{Re}, \mathrm{Os}$ as well as for $\left[M^{\mathrm{I}}\left(\mathrm{NH}_{3}\right)_{5} \mathrm{Cl}\right]\left[M^{\mathrm{II}} \Gamma_{4}\right]$ with $M^{\mathrm{I}}=\mathrm{Co}, \mathrm{Cr}, \mathrm{Ir}, \mathrm{Rh}$ and Os, $M^{\mathrm{II}}=\mathrm{Pt}$ and $\mathrm{Pd}, \Gamma=\mathrm{Cl}$ and $\mathrm{Br}$ were described for all possible metallic combinations [20,21]. Nevertheless, not all $\left[M^{\mathrm{I}}\left(\mathrm{NH}_{3}\right)_{5} \mathrm{Cl}\right]\left[M^{\mathrm{II}} \Gamma_{6}\right]$ compounds $M^{\mathrm{I}}=\mathrm{Co}, \mathrm{Cr}$, Ir, Rh and Os, $M^{\mathrm{II}}=\mathrm{Ir}$, Pt, Re and Os and $\Gamma=\mathrm{Cl}$ and $\mathrm{Br}$ were synthetized and structurally investigated so far. For $\left[\mathrm{M}^{\mathrm{I}}\left(\mathrm{NH}_{3}\right)_{5} \mathrm{Cl}\right]_{2}\left[\mathrm{M}^{\mathrm{II}} \mathrm{Cl}_{6}\right] \mathrm{Cl}_{2}$ and $\left[M^{\mathrm{I}}\left(\mathrm{NH}_{3}\right)_{5} \mathrm{Cl}\right]\left[M^{\mathrm{II}} \Gamma_{4}\right]$, it has been shown that isoformular compounds were isostructural and could be co-crystallized from water solutions. $\left[M^{\mathrm{I}}\left(\mathrm{NH}_{3}\right)_{5} \mathrm{Cl}\right]\left[\mathrm{M}^{\mathrm{II}} \mathrm{Cl}_{6}\right]$ salts were crystallized in the $P 2_{1} / m$ space group. All species were also isostructural with very close cell parameters.

$\left[M^{\mathrm{I}}\left(\mathrm{NH}_{3}\right)_{5} \mathrm{Cl}\right]\left[\mathrm{M}^{\mathrm{II}} \mathrm{Br}_{6}\right]$ compounds were synthetized only for selected metals. It has been shown that various metals in anion give various types of crystal structures. To clarify general trends in the isoformular compounds where not all members are isostructural is was important to extend them with other examples. In the current study, $\left[\operatorname{Ir}\left(\mathrm{NH}_{3}\right)_{5} \mathrm{Cl}\right]\left[\mathrm{PtBr}_{6}\right]$ salt was prepared as single crystals and its crystal structure was compared with isoformular $\left[\operatorname{Ir}\left(\mathrm{NH}_{3}\right)_{5} \mathrm{Cl}\right]\left[\mathrm{PtCl}_{6}\right],\left[\mathrm{Rh}_{(}\left(\mathrm{NH}_{3}\right)_{5} \mathrm{Cl}\right]\left[\mathrm{PtBr}_{6}\right]$, $\left[\operatorname{Ir}\left(\mathrm{NH}_{3}\right)_{5} \mathrm{Cl}\right]\left[\mathrm{PtBr}_{6}\right]$ and $\left[\mathrm{Rh}\left(\mathrm{NH}_{3}\right)_{5} \mathrm{Cl}\right]\left[\mathrm{IrBr}_{6}\right]$ and also previously structurally characterized by $\mathrm{X}$-ray diffraction as single crystals (Table 2).

Table 2. Crystallographic characteristics for $\left[M^{\mathrm{I}}\left(\mathrm{NH}_{3}\right)_{5} \mathrm{Cl}\right]\left[M^{\mathrm{II}} \Gamma_{6}\right] M^{\mathrm{I}}=\mathrm{Rh}$, Ir; $M^{\mathrm{II}}=\mathrm{Ir}, \mathrm{Pt}$ and $\Gamma=\mathrm{Cl}$ and $\mathrm{Br}$ single-source precursors.

\begin{tabular}{|c|c|c|c|c|c|}
\hline Composition & $\begin{array}{c}{\left[\mathrm{Ir}\left(\mathrm{NH}_{3}\right)_{5} \mathrm{Cl}\right]\left[\mathrm{PtCl}_{6}\right]} \\
\text { Powder, RT }\end{array}$ & $\begin{array}{c}{\left[\mathrm{Ir}\left(\mathrm{NH}_{3}\right)_{5} \mathrm{Cl}\right]\left[\mathrm{PtBr}_{6}\right]} \\
\text { Single Crystal, } 150 \\
\text { K }\end{array}$ & $\begin{array}{c}{\left[\mathrm{Rh}\left(\mathrm{NH}_{3}\right)_{5} \mathrm{Cl}\right]\left[\mathrm{IrCl}_{6}\right]} \\
\text { Powder, RT }\end{array}$ & 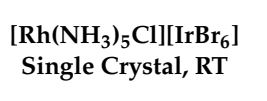 & 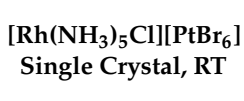 \\
\hline$a, \AA$ & $11.568(3)$ & 11.9099(13) & $11.67(6)$ & $12.030(6)$ & $12.013(2)$ \\
\hline$b, \AA$ & $8.314(2)$ & $8.3277(9)$ & $8.348(7)$ & $8.532(5)$ & $8.401(2)$ \\
\hline$c, \AA$ & $16.104(3)$ & $15.832(2)$ & $15.65(3)$ & $16.382(6)$ & $15.999(3)$ \\
\hline $\mathrm{b}^{\circ}$ & $110.15(5)$ & $90.000(4)$ & 105.7(3) & $106.23(1)$ & $91.13(3)$ \\
\hline$V, \AA^{3}$ & 1454.1 & 1570.3(3) & 1468.0 & 1614.4 & 1614.4 \\
\hline Space group & $P 2_{1} / m$ & $P 2_{1} / m$ & $P 2_{1} / m$ & $P 2_{1} / m$ & $P 2_{1} / m$ \\
\hline$Z$ & 4 & 4 & 4 & 4 & 4 \\
\hline $\begin{array}{c}\text { Molecular } \\
\text { weight }\end{array}$ & 720.62 & 987.32 & 628.45 & 895.15 & 898.01 \\
\hline$D, \mathrm{~g} / \mathrm{cm}^{3}$ & 3.287 & 4.176 & 2.844 & 3.683 & 3.70 \\
\hline PDF number & - & - & - & 00-057-086501-080-8875 & 01-072-8177 \\
\hline ICSD number & - & 1971298 & - & 421153 & 98115 \\
\hline Reference & present study & present study & [14] & [15] & [12] \\
\hline
\end{tabular}

All investigated isoformular double complex salts contain isolated chloropentammine cations (Rh and $\mathrm{Ir}$ ) with local symmetry $m$ with one $\mathrm{NH}_{3}$ and one $\mathrm{Cl}$ ligand sitting in the mirror plane. Hexachloroand hexabromometalates(IV) as anions had local symmetry $2 / m$ with four Cl-ligands placed in the mirror-plane. In all crystal structures, six anions have six neighboring cations as well as six cations had six neighboring anions. The coordination polyhedral for cations and anions was deformed octahedra, so general crystal structures can be described as deformed $\mathrm{NaCl}$ structures with closed packing of isometric cations and anions. Cations and anions form regular closed-packed hexagonal layers perpendicular to the direction [110].

Salts with hexachlorometallates(IV) as anions had relatively low solubility and were difficult in crystallization from water solution. Only parent compound $\left[\mathrm{Rh}\left(\mathrm{NH}_{3}\right)_{5} \mathrm{Cl}\right]\left[\mathrm{OsCl}_{6}\right]$ was crystallized as a single crystal (Figure 2) [14]. $\left[\mathrm{Ir}\left(\mathrm{NH}_{3}\right)_{5} \mathrm{Cl}\right]\left[\mathrm{PtCl}_{6}\right]$ was obtained only as crystalline powder. Its crystal structures were similar to isoformular $\left[\mathrm{Rh}\left(\mathrm{NH}_{3}\right)_{5} \mathrm{Cl}\right]\left[\mathrm{PtCl}_{6}\right]$ with close cell parameters.

Double complex salts with hexabromometallates(IV) as anions have usually a higher solubility and can be prepared as single crystals suitable for X-ray diffraction study [15]. Crystal structures of 
[ $\left.\mathrm{Rh}\left(\mathrm{NH}_{3}\right)_{5} \mathrm{Cl}\right]\left[\mathrm{IrBr}_{6}\right]$ and $\left[\mathrm{Rh}\left(\mathrm{NH}_{3}\right)_{5} \mathrm{Cl}\right]\left[\mathrm{PtBr}_{6}\right]$ were described in $[12,15]$ based on single crystal X-ray diffraction data. Salts with hexabromometallates(IV) as anions have monoclinic structures solved in similar to the haxachlorometallates(IV) $P 2_{1} / m$ space group. Nevertheless, monoclinic angle $\beta$ was close to $90^{\circ}$. [ $\left.\operatorname{Ir}\left(\mathrm{NH}_{3}\right)_{5} \mathrm{Cl}\right]\left[\mathrm{PtBr}_{6}\right]$ had the same crystal structure as $\left[\mathrm{Rh}\left(\mathrm{NH}_{3}\right)_{5} \mathrm{Cl}\right]\left[\mathrm{PtBr}_{6}\right]$ with a monoclinic angle much closer to $90^{\circ}$. Due to the monoclinic angle, all selected single crystals were twinned by $180^{\circ}$ rotation around the $c$ axis and might be indexed as orthorhombic. Diffraction reflections were much blurrier at higher angles. Nevertheless, its crystal structure was not orthorhombic and did not show higher symmetry. Tests for higher possible symmetry did not give positive results. The corresponding dataset for $\left[\operatorname{Ir}\left(\mathrm{NH}_{3}\right)_{5} \mathrm{Cl}\right]\left[\mathrm{PtBr}_{6}\right]$ was collected and integrated in the orthorhombic crystal system and later refined as a two component twin with $0.528 / 0.472$ twin fractions. The real value of the monoclinic angle was approximately $90.5^{\circ}$ at $150 \mathrm{~K}$ and $90.8^{\circ}$ at $90 \mathrm{~K}$ (estimated using 100 weak individual reflections at high diffraction angles).

Crystal structures of $\left[\operatorname{Ir}\left(\mathrm{NH}_{3}\right)_{5} \mathrm{Cl}\right]\left[\mathrm{PtBr}_{6}\right]$ and $\left[\mathrm{Rh}\left(\mathrm{NH}_{3}\right)_{5} \mathrm{Cl}\right]\left[\mathrm{PtBr}_{6}\right]$ were similar to chloride analogous. Hexagonal closed packed layers of cations and anions typical for chloride structures were broken due to the presence of several short $\mathrm{Br} \ldots \mathrm{Br}(3.681,3.831,3.838 \AA)$ and $\mathrm{Br} \ldots \mathrm{Cl}(3.533 \AA)$ contacts. In $\left[\operatorname{Ir}\left(\mathrm{NH}_{3}\right)_{5} \mathrm{Cl}\right]\left[\mathrm{PtBr}_{6}\right]$, similar short contacts could be also found. Such $\mathrm{Br} \ldots \mathrm{Br}$ contacts were responsible for the packing of anions in the crystal structure as it was shown for other structures with hexabromometallates (IV).

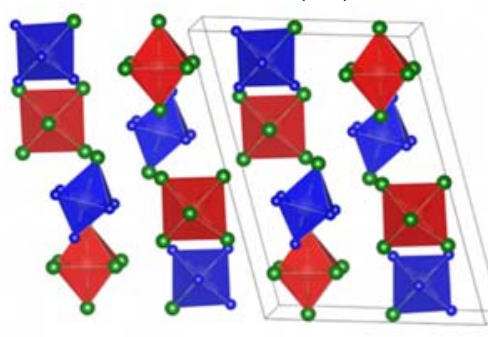

(a)

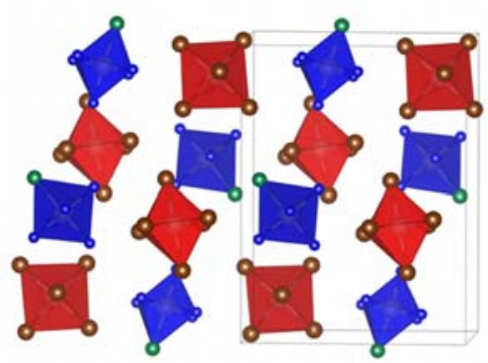

(b)

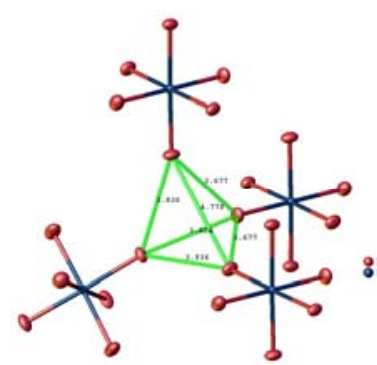

(c)

Figure 2. Crystal structure of $\left[\mathrm{Rh}\left(\mathrm{NH}_{3}\right)_{5} \mathrm{Cl}\right]\left[\mathrm{OsCl}_{6}\right]$ along the monoclinic axis y (a) [14]; right: crystal structure of $\left[\operatorname{Ir}\left(\mathrm{NH}_{3}\right)_{5} \mathrm{Cl}\right]\left[\mathrm{PtBr}_{6}\right]$ along the monoclinic axis $y$ and $(\mathbf{b})\left(\left[\mathrm{Rh}\left(\mathrm{NH}_{3}\right)_{5} \mathrm{Cll}\right]^{2+}\right.$ or $\left[\operatorname{Ir}\left(\mathrm{NH}_{3}\right)_{5} \mathrm{Cl}\right]^{2+}$ cations are shown in red, $\left[\mathrm{OsCl}_{6}\right]^{2-}$ or $\left[\mathrm{PtBr}_{6}\right]^{2-}$ anions and $\mathrm{N}-$ in blue, green $-\mathrm{Cl}$ or $\mathrm{Br} ; \mathrm{H}$ atoms are omitted for clarity); short $\mathrm{Br} \ldots \mathrm{Br}$ contacts between $\left[\mathrm{PtBr}_{6}\right]^{2-}$ anions in the $\left[\mathrm{Ir}\left(\mathrm{NH}_{3}\right)_{5} \mathrm{Cl}\right]\left[\mathrm{PtBr}_{6}\right]$ crystal structure (c).

\subsection{Preparation of fcc-Structured Binary Alloys under Ambient Pressure and Their Phase Composition}

All $f c c$-structured alloys were prepared from single-source precursors (Table 1). Among $f c c-$ structured binary metallic systems, only the Pt-Rh pair has complete miscibility in the solid state [22]. In all other binaries, there are miscibility gaps between two $f c c$-structured alloys: for Ir-Rh below $1335^{\circ} \mathrm{C}$; for Pd-Rh below $910^{\circ} \mathrm{C}$ and for Ir-Pt below $1370{ }^{\circ} \mathrm{C}$ [23-25]. Nevertheless, all single-source precursors gave single-phase alloys as products of their thermal decomposition in a reductive atmosphere even below $700{ }^{\circ} \mathrm{C}$ (Table 1). Thermal decomposition in an inert atmosphere usually results in a formation of two-phase mixtures, which might be a sign for different decomposition mechanisms.

All binary systems allow us to prepare various alloys by changing compositions of single-source precursors. So, crystallization of $\left[M^{\mathrm{I}}\left(\mathrm{NH}_{3}\right)_{5} \mathrm{Cl}\right]^{2+}\left(M^{\mathrm{I}}=\mathrm{Rh}\right.$, Ir) with $\left[M^{\mathrm{II}} \mathrm{Cl}_{6}\right]^{2-}\left(M^{\mathrm{II}}=\mathrm{Ir}\right.$, Pt $)$ from neutral water solutions results in a formation of $\left[M^{\mathrm{I}}\left(\mathrm{NH}_{3}\right)_{5} \mathrm{Cl}\right]\left[M^{\mathrm{II}} \mathrm{Cl}_{6}\right]$, which can be used as precursors for $f c c-M_{0.5}^{\mathrm{I}} M^{\mathrm{II}}{ }_{0.5}$ alloys: $\left[\mathrm{Rh}\left(\mathrm{NH}_{3}\right)_{5} \mathrm{Cl}\right]\left[\mathrm{IrCl}_{6}\right]$ for $f c c-\mathrm{Ir}_{0.5} \mathrm{Rh}_{0.5}$; $\left[\mathrm{Rh}\left(\mathrm{NH}_{3}\right)_{5} \mathrm{Cl}\right]\left[\mathrm{PtCl}_{6}\right]$ for $f c c-\mathrm{Rh}_{0.5} \mathrm{Pt}_{0.5}$; $\left[\operatorname{Ir}\left(\mathrm{NH}_{3}\right)_{5} \mathrm{Cl}\right]\left[\mathrm{PtCl}_{6}\right]$ for $f c c-\mathrm{Ir}_{0.5} \mathrm{Pt}_{0.5}$. Similar crystallization from $\mathrm{HCl}$-containing solutions results in a formation of $\left[M^{\mathrm{I}}\left(\mathrm{NH}_{3}\right)_{5} \mathrm{Cl}\right]_{2}\left[M^{\mathrm{II}} \mathrm{Cl}_{6}\right] \mathrm{Cl}_{2}$, which can be used as precursors for $f c c-M^{\mathrm{I}}{ }_{0.66} M^{\mathrm{II}}{ }_{0.33}$ alloys: $\left[\mathrm{Rh}\left(\mathrm{NH}_{3}\right)_{5} \mathrm{Cl}\right]_{2}\left[\mathrm{IrCl}_{6}\right] \mathrm{Cl}_{2}$ for $f c c-\mathrm{Ir}_{0.66} \mathrm{Rh}_{0.33} ;\left[\mathrm{Rh}\left(\mathrm{NH}_{3}\right)_{5} \mathrm{Cl}\right]_{2}\left[\mathrm{PtCl}_{6}\right] \mathrm{Cl}_{2}$ for $f c c-\mathrm{Rh}_{0.66} \mathrm{Pt}_{0.33}$ and $\left[\mathrm{Ir}\left(\mathrm{NH}_{3}\right)_{5} \mathrm{Cl}_{2}\left[\mathrm{PtCl}_{6}\right] \mathrm{Cl}_{2}\right.$ for $f c c-\mathrm{Ir}_{0.66} \mathrm{Pt}_{0.33}$. Only Ir-Pd and Rh-Pd systems allowed only a single 
type of precursors: $\left[\mathrm{Ir}\left(\mathrm{NH}_{3}\right)_{5} \mathrm{Cl}\right]\left[\mathrm{PdCl}_{4}\right]$ and $\left[\mathrm{Rh}\left(\mathrm{NH}_{3}\right)_{5} \mathrm{Cl}\right]\left[\mathrm{PdCl}_{4}\right]$ for $f c c-\mathrm{Ir}_{0.5} \mathrm{Pd}_{0.5}$ and $f c c-\mathrm{Rh}_{0.5} \mathrm{Pd}_{0.5}$ alloys respectively.

It seems that thermal decomposition of described systems could be controlled by a reaction atmosphere. In the reductive flow (hydrogen), all systems Ir-Rh, Pd-Rh, Ir-Pt and Pt-Rh formed single phase $f c c$-structured alloys. Single-phase alloys formed in systems with and without miscibility in the solid-state. In the inert atmosphere (argon or helium flow), Ir-Rh forms also a single-phase $f c c$-alloy [5]. Ir-Pt and Pt-Rh (both systems with miscibility in the solid-state) formed a two-phase mixture after heating in an inert flow, which might be due to the mechanism of their thermal decomposition.

In an inert atmosphere ( $\mathrm{He}, \mathrm{Ar}$ and $\mathrm{N}_{2}$ ), hexachlorometallates(IV) decompose in a relatively narrow temperature interval $[20,21]$. For $\left[\mathrm{Rh}\left(\mathrm{NH}_{3}\right)_{5} \mathrm{Cl}\right]\left[\mathrm{PtBr}_{6}\right]$ and $\left[\mathrm{Rh}\left(\mathrm{NH}_{3}\right)_{5} \mathrm{Cl}\right]\left[\mathrm{PtBr}_{4}\right][12,26]$, it has been shown that their thermal decomposition in an inert atmosphere corresponds to the formation of metallic $f c c-\mathrm{Pt}$ and $\mathrm{RhBr}_{3}$ as intermediates above $500^{\circ} \mathrm{C}$. Further heating results in the decomposition of $\mathrm{RhBr}_{3}$ and formation of two-phase $f c c$-alloys mixture. Such a transformation is a key process responsible for the formation of two-phase metallic products in an inert gas flow. Similarly, upon heating of $\left[\operatorname{Ir}\left(\mathrm{NH}_{3}\right)_{5} \mathrm{Cl}\right]\left[\mathrm{PtBr}_{6}\right]$ above $500^{\circ} \mathrm{C}$ intermediate with a total composition of " $\mathrm{Ir}: \mathrm{Pt}: \mathrm{Br}^{\prime}$ contains broad reflexes corresponding to $\mathrm{IrBr}_{3}$ and two $f(c-$-structured alloys:

$$
\left[\operatorname{Ir}\left(\mathrm{NH}_{3}\right)_{5} \mathrm{Cl}\right]\left[\mathrm{PtBr}_{6}\right] \rightarrow \operatorname{IrBr}_{3}+\text { "mixture of } f c c \text {-structured alloys". }
$$

Further heating results in the formation of a two-phase metallic mixture (Table 1). In general, thermal decomposition of $\left[M^{\mathrm{I}}\left(\mathrm{NH}_{3}\right)_{5} \mathrm{Cl}\right]\left[\mathrm{M}^{\mathrm{II}} \mathrm{Br}_{6}\right]$ occurred at higher temperatures in comparison with chloride $\left[\mathrm{M}^{\mathrm{I}}\left(\mathrm{NH}_{3}\right)_{5} \mathrm{Cl}\right]\left[\mathrm{M}^{\mathrm{II}} \mathrm{Cl}_{6}\right]$ analogous. Their thermal decomposition was overcome through the formation of rhodium or iridium bromides as intermediates, which was responsible for the formation of two-phase mixtures as final products of their thermal decomposition in an inert atmosphere. Thermal decomposition of $\left[\operatorname{Ir}\left(\mathrm{NH}_{3}\right)_{5} \mathrm{Cl}\right]\left[\mathrm{PtCl}_{6}\right]$ and $\left[\operatorname{Ir}\left(\mathrm{NH}_{3}\right)_{5} \mathrm{Cl}\right]\left[\mathrm{PtBr}_{6}\right]$ started at the same temperature (325 and $330^{\circ} \mathrm{C}$, correspondently), which could be due to the nature of their cation. Probably their thermal decomposition starts from the destruction of $\left[\operatorname{Ir}\left(\mathrm{NH}_{3}\right)_{5} \mathrm{Cl}\right]^{2+}$ cation.

Single-phase alloys prepared from single-source precursors show isometric porous metallic particles (Figure 3). The shape of porous conglomerates followed the shape of crystals characteristic for single source precursors.

A

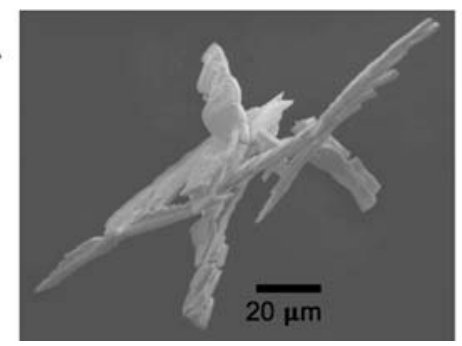

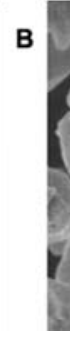

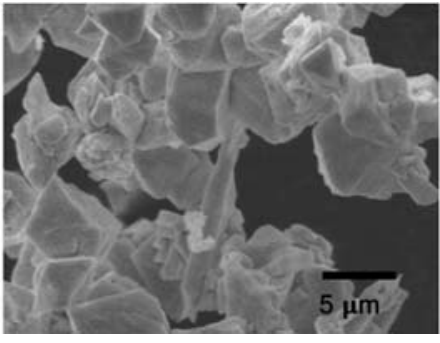

c

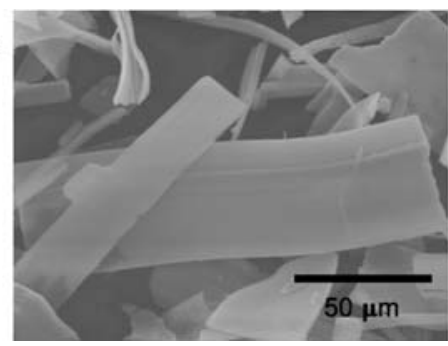

Figure 3. SEM images for $f c c-\operatorname{Ir}_{0.50} \mathrm{Pt}_{0.50}(\mathbf{A}), f c c-\mathrm{Rh}_{0.66} \mathrm{Pt}_{0.33}(\mathbf{B})$ and $f c c-\mathrm{Rh}_{0.50} \mathrm{Pd}_{0.50}$ (C) binary alloys.

\subsection{High-Pressure Compressibility of fcc-Structured Binary Refractory Alloys}

Cell parameters characteristic for prepared $f c c$-alloys correspond to Zen's low $[27,28]$ and nearly linearly depend on the alloy's compositions (Figure 4). Within experimental errors there is no positive or negative deviation from linearity, which might be a sign for ideality of described $f c c$-structured binary alloys. For $f c c$-structured binary alloys (and also for $h c p$-structured) with $h c p$ metals such as Ir-Re and Rh-Re alloys, significant negative deviation from linearity has been mentioned [29,30]. 


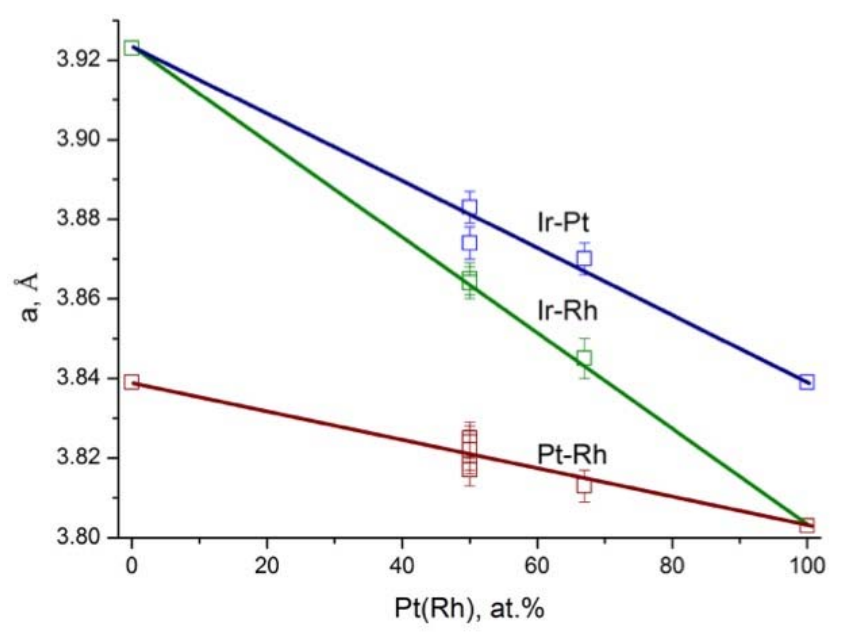

Figure 4. Dependence of cell parameters on composition for $f c c$-structured $\mathrm{Rh}, \mathrm{Ir}, \mathrm{Pt}$ and $\mathrm{Pd}$ single-phase binary alloys (according to Table 1).

All investigated $f c c$-structured refractory alloys do not show any pressure-induced phase transitions below $50 \mathrm{GPa}$ at room temperature. A similar pressure temperature stability was obtained for pure $\mathrm{Rh}, \mathrm{Ir}, \mathrm{Pd}$ and $\mathrm{Pt}$ up to much higher pressures. Experimental compressibility curves ( $P-V$ data) for all investigated alloys can be fitted using the third-order Birch-Murnaghan equation of state (BM-EoS) [31,32] (Table 3, Figure 5):

$$
P(V)=\frac{3 B_{0}}{2}\left[\left(\frac{V_{0}}{V}\right)^{\frac{7}{3}}-\left(\frac{V_{0}}{V}\right)^{\frac{5}{3}}\right]\left\{1+\frac{3}{4}\left(B_{0}^{\prime}-4\right)\left[\left(\frac{V_{0}}{V}\right)^{\frac{2}{3}}-1\right]\right\},
$$

where $V_{0}$ is the unit cell volume at ambient pressure, $B_{0}$ is the bulk modulus and $B_{0}^{\prime}$ is the pressure derivative of the bulk modulus. All alloys show regular compressibility with pressure as well as with composition.

It has been previously shown that bulk moduli for binary alloys can be estimated using quite a simple model reported in $[5,6,8]$. Concentration dependence of the bulk modulus $B_{0}(x)$ of a binary metallic alloy $M^{1}{ }_{x} M^{2}{ }_{1-x}$ containing $x$ atomic fraction of the refractory metal can be calculated using the following equation:

$$
B_{0}(x)=B_{2}\left[\frac{1+x\left(\frac{V_{1}}{V_{2}}-1\right)}{1+x\left(\frac{B_{2} V_{1}}{B_{1} V_{2}}-1\right)}\right]
$$

where $B_{1}$ and $B_{2}(\mathrm{GPa})$ are the bulk moduli of the metals $M^{1}$ and $M^{2}$, and $V_{1}$ and $V_{2}\left(\AA^{3}\right)$ are atomic volumes at ambient pressure of $M^{1}$ and $M^{2}$, correspondently. According to Table 3 , structural parameters (atomic volume and bulk moduli) for all alloys can be estimated quite well using simple models typical for ideal solid solutions. Such a finding can be used for a prediction of the compressibility of new alloys to be able to construct a complete thermodynamic database for refractory fcc-alloys.

Palladium shows relatively high compressibility in the comparison with the other platinum group metals. As a result, the $f c c-\mathrm{Rh}_{0.50} \mathrm{Pd}_{0.50}$ alloy had the highest compressibility among other alloys. At the same time its compressibility had the better accordance with the ideal solution model (Equation (2)). A similar good satisfaction between experimentally obtained data and predicted according to Equation (2) were found for the $f c c-\mathrm{Ir}_{0.42} \mathrm{Rh}_{0.58}$ alloy. Both investigated platinum alloys $f c c-\mathrm{Ir}_{0.50} \mathrm{Pt}_{0.50}$ and $f c c-\mathrm{Pt}_{0.33} \mathrm{Rh}_{0.67}$ show large deviations from predicted values. A larger value for $f c c-\mathrm{Ir}_{0.50} \mathrm{Pt}_{0.50}$ can be explained by relatively low experimental pressure. Nevertheless, compressibility of $f c c-\mathrm{Pt}_{0.33} \mathrm{Rh}_{0.67}$ was investigated up to $47 \mathrm{GPa}$. Its compressibility was much higher in comparison with ideal solutions model. The mentioned large deviation from the ideal solutions model should be further studied theoretically. 
Single-phase refractory high-entropy alloys, namely $h c p-\mathrm{Ir}_{0.19} \mathrm{Os}_{0.22} \mathrm{Re}_{0.21} \mathrm{Rh}_{0.20} \mathrm{Ru}_{0.19}$ and $f c c-\mathrm{Ir}_{0.26} \mathrm{Os}_{0.05} \mathrm{Pt}_{0.31} \mathrm{Rh}_{0.23} \mathrm{Ru}_{0.15}$, prepared from single-source precursors show similar numbers for atomic volumes and room-temperature compressibility (Table 3 ). Their behavior suggests that they can be described as ideal solid solutions [1,5]. Prepared $f c c$-structured binary alloys can be used as reliable models for modeling thermodynamic and structural properties of high-entropy alloys in a broad range of compositions. Phase instability upon heating and compression typical for high-entropy alloys with light metals such as $\mathrm{Al}, \mathrm{Co}, \mathrm{Ni}$ and $\mathrm{Fe}$ is not typical for refractory alloys based on platinum group metals. Platinum group metals are known as stable substances upon heating and compression. As soon as pure platinum metals and their binary alloys show extraordinary phase stability, their multicomponent alloys as well as high-entropy alloys did not show any phase transitions upon heating and compression, which makes them unique for high-temperature applications under extreme chemical impact and mechanical stress.

Table 3. Parameters of the equations of state (EOS) for fcc-structured Ir, Rh, Pt and Pd binary alloys and their high-entropy alloys.

\begin{tabular}{|c|c|c|c|c|c|}
\hline $\begin{array}{l}\text { Composition } \\
(\max . P)\end{array}$ & $\begin{array}{l}V_{0} / Z, \AA^{3} \cdot \text { atom }^{-1} \\
\quad\left(P=1 \text { bar }^{\mathrm{b}}\right.\end{array}$ & $\begin{array}{c}V_{0} / Z, \AA^{3} \cdot \text { atom }^{-1} \\
\text { According to } \\
\text { Zen's Rule }\end{array}$ & $\begin{array}{c}B_{0}, \mathrm{GPa} \\
B_{0}{ }^{\prime}\end{array}$ & $\begin{array}{c}B_{0}, \mathrm{GPa} \\
\text { According to } \\
\text { Equation (2) }\end{array}$ & Ref. \\
\hline $\begin{array}{l}f_{c c}-\mathrm{Ir}_{0.42} \mathrm{Rh}_{0.58} \\
\text { (up to } 57 \mathrm{GPa} \text { ) }\end{array}$ & $13.90(8)$ & 13.909 & $\begin{array}{l}317(17) \\
6.0(5)\end{array}$ & 316.9 & [5] \\
\hline $\begin{array}{l}f_{c c}-\mathrm{Ir}_{0.50} \mathrm{Pt}_{0.50} \\
\text { (up to } 15 \mathrm{GPa} \text { ) }\end{array}$ & $14.597(6)$ & 14.625 & $\begin{array}{c}321(6) \\
6(1)\end{array}$ & 304.7 & Sample A \\
\hline $\begin{array}{l}f_{c c}-\mathrm{Pd}_{0.50} \mathrm{Rh}_{0.50} \\
\text { (up to } 45 \mathrm{GPa} \text { ) }\end{array}$ & $14.18(2)$ & 14.224 & $\begin{array}{l}223(4) \\
5.0(3)\end{array}$ & 225.7 & Sample C \\
\hline $\begin{array}{l}f_{c c}-\mathrm{Pt}_{0.33} \mathrm{Rh}_{0.67} \\
\text { (up to } 47 \mathrm{GPa} \text { ) }\end{array}$ & $14.211(3)$ & 14.180 & $\begin{array}{l}259(1) \\
6.66(9)\end{array}$ & 292.1 & Sample B \\
\hline $\begin{array}{c}f(c c-\text { Ir } \\
\text { (up to } 67 \mathrm{GPa} \text { ) }\end{array}$ & $14.14(6)$ & - & $\begin{array}{l}341(10) \\
4.7(3)\end{array}$ & - & [5] \\
\hline $\begin{array}{c}f c c-R h \\
\text { (up to } 64 \mathrm{GPa} \text { ) }\end{array}$ & $13.73(7)$ & - & $\begin{array}{l}301(9) \\
3.1(2)\end{array}$ & - & [5] \\
\hline $\begin{array}{c}f_{c c-}-\mathrm{Pt} \\
\text { (up to } 100 \mathrm{GPa} \text { ) }\end{array}$ & $15.094(2)$ & - & $\begin{array}{l}277(2) \\
4.95(2)\end{array}$ & - & [33] \\
\hline $\begin{array}{c}f c c-\mathrm{Pd} \\
\text { (up to } 100 \mathrm{GPa} \text { ) }\end{array}$ & $14.718(2)$ & - & $\begin{array}{l}183 \\
5.28\end{array}$ & - & [34] \\
\hline $\begin{array}{c}f_{c c}-\mathrm{Ir}_{0.26} \mathrm{Os}_{0.05} \mathrm{Pt}_{0.31} \mathrm{Rh}_{0.23} \mathrm{Ru}_{0.15} \\
\text { (up to } 49 \mathrm{GPa} \text { ) }\end{array}$ & $14.16(9)$ & 14.262 & $\begin{array}{l}300(22) \\
6(1)\end{array}$ & - & [5] \\
\hline $\begin{array}{l}h c p-\operatorname{Ir}_{0.24} \mathrm{Os}_{0.21} \operatorname{Re}_{0.16} \mathrm{Rh}_{0.18} \mathrm{Ru}_{0.20} \\
\text { (up to } 45 \mathrm{GPa} \text { ) }\end{array}$ & $13.979(2)$ & 13.882 & $\begin{array}{l}317(2) \\
4.9(1)\end{array}$ & - & [1] \\
\hline
\end{tabular}

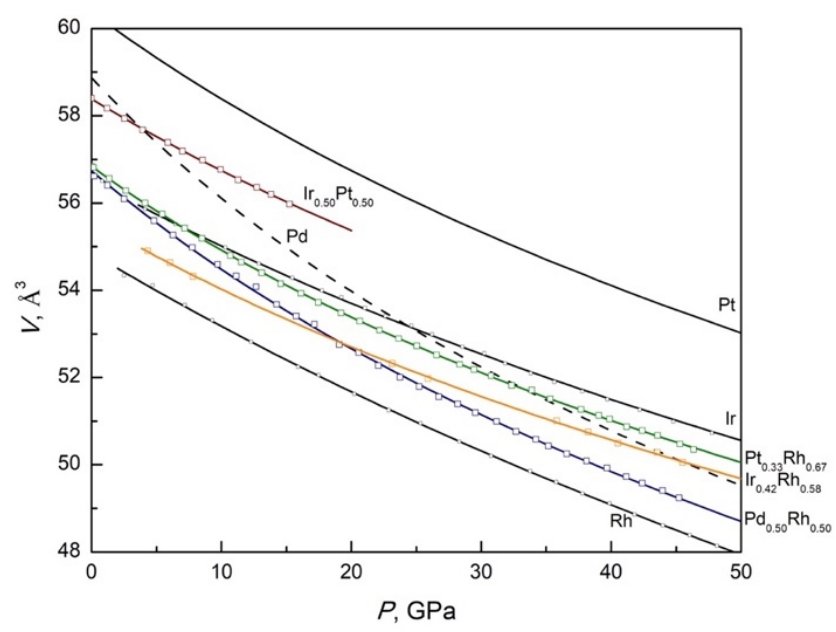

Figure 5. Room temperature high-pressure compressibility curves for $f c c$-structured $\mathrm{Rh}, \mathrm{Ir}$, Pt and Pd binary alloys and pure metals in $V / Z$ vs. $P$ scale (according to Table 3 ). 


\section{Conclusions}

Single-source precursors strategy can be successfully applied for the preparation of high-entropy alloys of various compositions and structures. Double complex salts can be considered as effective single-source precursors for refractory multicomponent alloys. A systematic investigation of single-source precursors for binary alloys gives experimental evidence for synthetic possibilities to access multicomponent systems. Refractory alloys prepared using single-source precursors can be further applied as active elements of catalytic reactors, electrochemical and fuel cells $[1,8,35,36]$. Diverse single-source precursors of various chemical functionality, composition and stability should be designed to prepare useful functional alloys as nanostructured powders for catalytic applications. Based on the materials presented we could conclude the following:

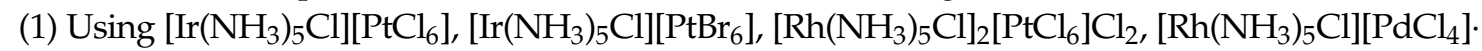
$\mathrm{H}_{2} \mathrm{O}$ and $\left[\mathrm{Rh}\left(\mathrm{NH}_{3}\right)_{5} \mathrm{Cl}\right]\left[\mathrm{IrCl}_{6}\right]$ as single-source precursors, $f\left(c-\mathrm{Ir}_{0.509(5)} \mathrm{Pt}_{0.491(5)}, f c c-\mathrm{Rh}_{0.657(4)} \mathrm{Pt}_{0.343(4)}\right.$, and $f c c-\mathrm{Rh}_{0.497(5)} \mathrm{Pd}_{0.502(5)}$ could be prepared by thermal decomposition in a hydrogen flow below $800{ }^{\circ} \mathrm{C}$.

(2) Only $f_{c c}-\mathrm{Rh}_{0.66} \mathrm{Pt}_{0.33}$ corresponded to the single-phase region on the phase diagram. $F c c-\mathrm{Ir}_{0.50} \mathrm{Pt}_{0.50}, f c c-\mathrm{Rh}_{0.50} \mathrm{Pd}_{0.50}$ and $f c c-\mathrm{Rh}_{0.50} \mathrm{Ir}_{0.50}$ alloys corresponded to miscibility gaps on binary phase diagrams.

(3) Crystal structure and thermal decomposition in an inert atmosphere of bromide-containing salt $\left[\operatorname{Ir}\left(\mathrm{NH}_{3}\right)_{5} \mathrm{Cl}\right]\left[\mathrm{PtBr}_{6}\right]$ were significantly different in comparison with isoformular chloride-based $\left[\operatorname{Ir}\left(\mathrm{NH}_{3}\right)_{5} \mathrm{Cl}\right]\left[\mathrm{PtCl}_{6}\right]$. Its thermal decomposition occurred at higher temperature with a formation of $\mathrm{IrBr}_{3}$ as a possible intermediate phase.

(4) Room temperature compression of $f_{c}-\mathrm{Ir}_{0.50} \mathrm{Pt}_{0.50}, f c c-\mathrm{Rh}_{0.66} \mathrm{Pt}_{0.33}, f_{c} c-\mathrm{Rh}_{0.50} \mathrm{Pd}_{0.50}$ and $f c c-\mathrm{Rh}_{0.50} \mathrm{Ir}_{0.50}$ alloys up to $50 \mathrm{GPa}$ did not reveal any phase transitions. Compressibility curves can be fitted using the third-order Birch-Murnaghan equation of state. For $f c c-\operatorname{Ir}_{0.50} \mathrm{Pt}_{0.50}, V_{0} / Z=$ 14.597(6) $\AA^{3} \cdot$ atom $^{-1}, B_{0}=321(6) \mathrm{GPa}$ and $B_{0}{ }^{\prime}=6(1)$; for $f c c-\mathrm{Rh}_{0.66} \mathrm{Pt}_{0.33}, V_{0} / Z=14.211(3) \AA^{3} \cdot \mathrm{atom}^{-1}$, $B_{0}=259(1) \mathrm{GPa}$ and $B_{0}{ }^{\prime}=6.66(9)$ and for $f c c-\mathrm{Rh}_{0.50} \mathrm{Pd}_{0.50}, V_{0} / Z=14.18(2) \AA^{3} \cdot \mathrm{atom}^{-1}, B_{0}=223(4) \mathrm{GPa}$ and $B_{0}{ }^{\prime}=5.0(3)$.

Author Contributions: Conceptualization, K.V.Y., L.S.D. and S.A.G.; Crystal structure solution and refinement, A.S.; High-pressure study, Y.K.V., S.K., M.B., T.F., M.H.; all authors contributed to writing and editing manuscript. All authors have read and agreed to the published version of the manuscript.

Funding: This research received no external funding.

Acknowledgments: The authors thank the ID15B beamline at the European Synchrotron Radiation Facility, Grenoble, France, for providing us with measurement time and technical support. Ines Feldmann (BAM) is thanked for the collection of SEM/EDX images.

Conflicts of Interest: The authors declare no conflict of interest.

\section{References}

1. Yusenko, K.V.; Riva, S.; Carvalho, P.A.; Yusenko, M.V.; Arnaboldi, S.; Sukhikh, A.; Hanfland, M.; Gromilov, S.A. First hcp HEA with outstanding stability under extreme conditions and high electrocatalytic activity in methanol oxidation. Scripta Materialia 2017, 138, 22-27. [CrossRef]

2. Occelli, F.; Farber, D.L.; Badro, J.; Aracne, C.M.; Teter, D.M.; Hanfland, M.; Canny, B.; Couzinet, B. Experimental evidence for a high-pressure isostructural phase transition in osmium. Phys. Rev. Lett. 2004, 93, 095502. [CrossRef] [PubMed]

3. Dubrovinsky, L.; Dubrovinskaia, N.; Prakapenka, V.B.; Abakumov, A.M. Implementation of micro-ball nanodiamond anvils for high-pressure studies above 6 Mbar. Nat. Commun. 2012, 3, 1-7. [CrossRef] [PubMed]

4. Dubrovinsky, L.; Dubrovinskaia, N.; Bykova, E.; Bykov, M.; Prakapenka, V.; Prescher, C.; Glazyrin, K.; Liermann, H.-P.; Hanfland, M.; Ekholm, M.; et al. The most incompressible metal osmium at static pressures above 750 gigapascals. Nature 2015, 525, 226-229. [CrossRef] 
5. Yusenko, K.V.; Khandarkhaeva, S.; Fedotenko, T.; Pakhomova, A.; Gromilov, S.A.; Dubrovinsky, L.; Dubrovinskaia, N. Equations of state of Rhodium, Iridium and their alloys up to 70 GPa. J. Alloy. Comp. 2019, 788, 212-218. [CrossRef]

6. Yusenko, K.V.; Bykova, E.; Bykov, M.; Gromilov, S.A.; Kurnosov, A.V.; Prescher, C.; Prakapenka, V.B.; Hanfland, M.; van Smaalen, S.; Margadonna, S.; et al. Compressibility of Ir-Os alloys under high pressure. J. Alloy. Comp. 2015, 622, 155-161. [CrossRef]

7. Yusenko, K.V.; Bykova, E.; Bykov, M.; Gromilov, S.A.; Kurnosov, A.V.; Prescher, C.; Prakapenka, V.B.; Crichton, W.A.; Hanfland, M.; Margadonna, S.; et al. High-pressure high-temperature stability of $h c p-\operatorname{Ir}_{x} \mathrm{Os}_{1-x}$ ( $x=0.50$ and 0.55) alloys. J. Alloy. Comp. 2017, 700, 198-207. [CrossRef]

8. Yusenko, K.V.; Bykova, E.; Bykov, M.; Riva, S.; Crichton, W.A.; Yusenko, M.V.; Sukhikh, A.S.; Arnaboldi, S.; Hanfland, M.; Dubrovinsky, L.S.; et al. Ir-Re binary alloys under extreme conditions and their electrocatalytic activity in methanol oxidation. Acta Mater. 2017, 139, 236-243. [CrossRef]

9. Shubin, Y.V.; Korenev, S.V.; Yusenko, K.V.; Korda, T.M.; Venediktov, A.B. X-ray study of double complex salts $\left[M\left(\mathrm{NH}_{3}\right)_{5} \mathrm{Cl}\right]\left[M^{*} \mathrm{Cl}_{4}\right]$ - precursors of polymetallic powders $\left(M=\mathrm{Ir}, \mathrm{Rh}, \mathrm{Co} ; M^{*}=\mathrm{Pt}, \mathrm{Pt}\right)$. Russ. Chem. Bull. 2002, 51, 41-45. [CrossRef]

10. Bykov, M.; Yusenko, K.V.; Bykova, E.; Pakhomova, A.; Kraus, W.; Dubrovinskaia, N.; Dubrovinsky, L. Synthesis of arsenopyrite-type rhodium pernitride $\mathrm{RhN}_{2}$ from a single-source azide precursor. Eur. J. Inorg. Chem. 2019, 32, 3667-3671. [CrossRef]

11. Garnier, E. Structure of Bis[pentaamminechloroiridium(III)] Hexachloroplatinate(IV) Dicloride. Acta Cryst. C. 1993, C49, 578-580. [CrossRef]

12. Yusenko, K.V.; Gromilov, S.A.; Baidina, I.A.; Shubin, Y.V.; Korolkov, I.V.; Drebushchak, T.N.; Basova, T.V.; Korenev, S.V. Synthesis, structure, and thermal decomposition of chloropentamminerhodium(III) hexabromoplatinate(IV). J. Struct. Chem. 2002, 43, 649-655. [CrossRef]

13. Yusenko, K.V.; Gromilov, S.A.; Korenev, S.V.; Baidina, I.A.; Korolkov, I.V.; Drebushchak, T.N. Synthesis and crystal structure of $\left[\mathrm{Rh}\left(\mathrm{NH}_{3}\right)_{5} \mathrm{Cl}_{2}\left[\mathrm{PtCl}_{6}\right] \mathrm{Cl}_{2}\right.$. J. Struct. Chem. 2002, 43, 697-699. [CrossRef]

14. Gromilov, S.A.; Korenev, S.V.; Baidina, I.A.; Korolkov, I.V.; Yusenko, K.V. Syntheses of $\left[\mathrm{Rh}\left(\mathrm{NH}_{3}\right)_{5} \mathrm{Cl}\right]\left[\mathrm{MCl}_{6}\right]$ $(M=\mathrm{Re}, \mathrm{Os}, \mathrm{Ir})$ and investigation of their thermolysis products. Crystal structure of $\left[\mathrm{Rh}\left(\mathrm{NH}_{3}\right)_{5} \mathrm{Cl}\right]\left[\mathrm{OsCl}_{6}\right] . J$. Struct. Chem. 2002, 43, 488-494. [CrossRef]

15. Yusenko, K.V.; Shusharina, E.A.; Gromilov, S.A. X-ray diffraction study of $\left[M^{\mathrm{I}}\left(\mathrm{NH}_{3}\right)_{5}\right]\left[M^{\mathrm{II}} \mathrm{Br}_{6}\right]\left(M^{\mathrm{I}}=\mathrm{Rh}\right.$, Ir; $M^{\mathrm{II}}=\mathrm{Re}$, Ir) polycrystals. J. Struct. Chem. 2010, 51, 935-941. [CrossRef]

16. Yusenko, K.V.; Gromilov, S.A.; Baidina, I.A.; Korolkov, I.V.; Zhivonitko, V.V.; Venediktov, A.B.; Korenev, S.V. Crystal structure of $\left[M\left(\mathrm{NH}_{3}\right)_{5} \mathrm{Cl}_{2}\left[\mathrm{IrCl}_{6}\right] \mathrm{Cl}_{2}(M=\mathrm{Co}\right.$, Rh, Ir) binary complex salts. J. Struct. Chem. 2003, 44, 60-67. [CrossRef]

17. Prescher, C.; Prakapenka, V.B. DIOPTAS: A program for reduction of two-dimensional X-ray diffraction data and data exploration. High Press. Res. 2015, 35, 223-230. [CrossRef]

18. Petříček, V.; Dušek, M.; Palatinus, L. Crystallographic computing system JANA2006: General Features. Z. Krist 2014, 229, 345-352. [CrossRef]

19. Angel, R.J. Equations of State. In Hazen, R.M., Downs, R.T. (Eds.). High-pressure, high-temperature crystal chemistry. Rev. Mineral. Geochem. 2001, 41, 35-60. [CrossRef]

20. Korenev, S.V.; Venediktov, A.B.; Shubin, Y.V.; Gromilov, S.A.; Yusenko, K.V. Synthesis and structure of binary complexes of platinum group metals - precursors of metallic materials. J. Struct. Chem. 2003, 44, 46-59. [CrossRef]

21. Pechenyuk, S.I.; Domonov, D.P. Properties of binary complex compounds. J Struct. Chem. 2011, 52, $412-427$. [CrossRef]

22. Darling, A.S.; Mech, A.M.I. Rhodium Platinum Alloys. A Critical Review of Their Constitution and Properties. Platin. Met. Rev. 1961, 5, 58-65.

23. Tripathi, S.N.; Bharadwaj, S.R.; Chandrasekharaia, M.S. The Ir-Rh (Iridium—Rhodium) system. J. Phase Equilibria 1991, 12, 606-608. [CrossRef]

24. Gürler, R.; Cornish, L.A.; Pratt, J.N. Computer assessment of the palladium-rhodium system. J. Alloys Comp. 1993, 191, 165-168. [CrossRef]

25. Yamabe-Mitarai, Y.; Aoyagi, T.; Abe, T. An investigation of phase separation in the Ir-Pt binary system. J. Alloys Comp. 2009, 484, 327-334. [CrossRef] 
26. Venediktov, A.B.; Korenev, S.V.; Shubin, Y.V.; Kuznetsov, N.A.; Yusenko, K.V. Synthesis and properties of double complexes $\left[\mathrm{M}\left(\mathrm{NH}_{3}\right)_{5} \mathrm{Cl}\right]\left[\mathrm{PdBr}_{4}\right](M=\mathrm{Co}$, Rh, Ir). Russ. J. Inorg. Chem. 2003, 48, 379-384.

27. Zen E-an. Validity of "Vegard's Law". Amer. Min. 1956, 41, 523-524.

28. Denton, A.R.; Ashcroft, N.W. Vegard's Law. Phys. Rev. A 1991, 43, 3161. [CrossRef]

29. Yusenko, K.V. Phase diagram of the iridium—rhenium metallic system. Platin. Met. Rev. 2013, 57, 57-65. [CrossRef]

30. Yusenko, K.V. Phase diagram of the rhenium_rhodium system: State of the art. Platin. Met. Rev. 2011, 54, 188-194. [CrossRef]

31. Varotsos, P.; Alexopoulos, K. Series, Defects in solids. In Thermodynamics of point defects and their relation with bulk properties; Amelinckx, S., Gevers, R., Nihoul, J., Eds.; North-Holland Publ. Co.: Amsterdam, The Netherlands, 1986; pp. 156-158, 325-347.

32. Introduction to the physics of the Earth's interior. Available online: http://www.doganaydal.com/nesneler/ kutuphanekitaplar/INTRODUCTION_TO_THE_PHYSICS_OF_THE_EARTHS_INTERIOR.PDF (accessed on 9 January 2020).

33. Yingwei, F.; Ricolleau, A.; Frank, M.; Mibe, K.; Shen, G.; Prakapenka, V. Toward an internally consistent pressure scale. PNAS 2007, 104, 9182-9186.

34. Mao, H.K.; Bell, P.M.; Shaner, J.W.; Steinberg, D.J. Specific volume measurements of Cu, Mo, Pd, and Ag and calibration of the ruby R1 fluorescence pressure gauge from 0.06 to 1 Mbar. J. Appl. Phys. 1978, 49, 3276-3283. [CrossRef]

35. Domonov, D.P.; Pechenyuk, S.I.; Belyaevskii, A.T.; Yusenko, K.V. Formation of Nanostructured Carbon from $\left[\mathrm{Ni}\left(\mathrm{NH}_{3}\right)_{6}\right]_{3}\left[\mathrm{Fe}(\mathrm{CN})_{6}\right]_{2}$. Nanomaterials 2020, 10, 389. [CrossRef] [PubMed]

36. Domonov, D.P.; Pechenyuk, S.I.; Semushina, Y.P.; Yusenko, K.V. Solid-state transformations in inner coordination sphere of $\left[\mathrm{Co}\left(\mathrm{NH}_{3}\right)_{6}\right]\left[\mathrm{Fe}\left(\mathrm{C}_{2} \mathrm{O}_{4}\right)_{3}\right] \cdot 3 \mathrm{H}_{2} \mathrm{O}$ as a route to access catalytically active Co-Fe materials. Materials 2019, 12, 221. [CrossRef]

(C) 2020 by the authors. Licensee MDPI, Basel, Switzerland. This article is an open access article distributed under the terms and conditions of the Creative Commons Attribution (CC BY) license (http://creativecommons.org/licenses/by/4.0/). 